\title{
Experimental Study on Dynamic Characteristics of Shut-In and Restart Process in Water Drive Strong Heterogeneous Oil Reservoir
}

\author{
Hao Chen $\mathbb{D}^{1,2}{ }^{1,2}$ Mingyang Yang, ${ }^{1}$ Haizeng Yu, ${ }^{1}$ Shenglai Yang, ${ }^{1,2}$ and Mibang Wang ${ }^{1}$ \\ ${ }^{1}$ Key Lab of Petroleum Eng. of MOE, China University of Petroleum, Beijing, China 102249 \\ ${ }^{2}$ State Key Laboratory of Petroleum Resources and Engineering, Beijing, China 102249 \\ Correspondence should be addressed to Hao Chen; chenhao@cup.edu.cn
}

Received 13 November 2020; Revised 16 March 2021; Accepted 22 March 2021; Published 14 April 2021

Academic Editor: Meng Meng

Copyright (C) 2021 Hao Chen et al. This is an open access article distributed under the Creative Commons Attribution License, which permits unrestricted use, distribution, and reproduction in any medium, provided the original work is properly cited.

\begin{abstract}
The main purpose of this investigation is to study the dynamic characteristics of shut-in and restart process in reservoirs with high water cut and strong vertical heterogeneity. The physical model, which includes three layers with low, medium, and highpermeability from top to bottom, was made according to the similarity law. Water drive test, the first restart test, and the second restart test were conducted, respectively. Water cut, oil recovery, and saturation distribution of the remaining oil were obtained during the tests. On this basis, mechanisms of shut-in and restart process of the reservoir were analyzed. It is concluded that appropriate developing plan such as layering mining and cyclic waterflooding should be implemented for developing strong heterogeneity reservoirs. The shut-in and restart tests showed that closing the water-flooded layer is beneficial for enlarging the sweep volume. Besides, water cut of $98 \%$ does not mean the economic limits of waterflooding. Under the effect of capillary force and gravitational differentiation, oil and water will redistribute in the formation. The redistribution of the oil and water, the fluctuation of the pressure difference, and the rebuild of the flow path, which produce parts of the bypassed oil, are the main mechanisms of the recovery enhancement by shut-in and restart operation. It should be noted that the shut-in and restart process indeed prolongs the waterflooding development. However, simply replying on the oil and water distribution under static conditions cannot greatly enhance the oil recovery.
\end{abstract}

\section{Introduction}

Water flooding is one of the main methods for enhancing oil recovery after depletion drive. Due to the advantages of low pollution to the environment and low damage to the formation, it is widely applied in the oilfields all over the world [1, $2]$. For some reservoirs with good geological conditions (permeability is more than $100 \times 10^{-3} \mu \mathrm{m}^{2}$, reservoir porosity is greater than $15 \%$, no phenomenon of water sensitivity, etc.), water flooding can increase recovery of over $60 \%$. However, due to the complexity and heterogeneity, phenomena such as fingering, tonguing, and water channeling are very common in most reservoirs. The reduction of the sweep efficiency greatly decreases the final oil recovery. Typically, when the water cut of the production well reaches $98 \%$, the actual oil recovery is only $20 \%-40 \%$ by the way of waterflood- ing. For these reservoirs entering high water cut stage, how to enhance the waterflooding recovery is one of the key issues for the petroleum engineers $[3,4]$.

Tertiary oil recovery, including miscible flooding, chemical flooding, and thermal flooding, is a good way to enhance the waterflooding recovery. It is reported that the cumulative oil recovery attains about $60 \%-80 \%[5,6]$. However, for an oilfield with steady, economical, and long-term development plan, water flooding is still one of the most potential recovery methods [7]. Thus, extensive research has been conducted on how to improve the waterflooding effect $[8,9]$.

Generally, there are two main kinds of methods to enhance the waterflooding development effect. One is readjustment of injection and production pattern, profile control and water shut-off, and slicing mining [10-15]. Another one is to change the working schedule, namely adopting different 
water injection patterns based on the formation characteristics. The main injection pattern includes alternating water injection, alternating injection and production, and intermittent injection and production [16-18]. Unstable water injection can improve the water displacement effect by changing the water injection and production rate cyclically. An unsteady pressure field was built, and the fluid was redistributed in the formation. Because of the pressure drops between the layers, the interlayer flow occurs and capillary imbibition effect strengthens, which finally enhance the sweep efficiency. According to the stages, it can be referred to as cyclic water injection, impulsive water injection, and intermittent injection [19-21].

Cyclic water injection is a water injection method which changes the working schedule of the oil and water wells regularly based on the existing well pattern. The injection patterns of the well groups as the unit are varied alternately. An unsteady pressure drop was built in the formation which brings the unswept regions back into development and then enhances the sweep efficiency of the heterogeneity formation. It is widely used in the strong heterogeneity reservoir. The coupling effect of cyclic injection and fluid division brings the water into lower permeability layers. It also creates favorable conditions for the cross-flow between the layers of lower permeability and higher permeability $[22,23]$. Cyclic water flooding is mostly better than conventional water flooding, typically $1 \%-10 \%$ higher in oil recovery. The main characteristics of cyclic water flooding are as follows: (1) the increase in recovery rate decreases as the degree of water flooding deepens; (2) if the heterogeneity for the reservoir is strong, the effect of the cyclic water flooding is important; (3) the earlier the cyclic water flooding, the higher increase in oil recovery $[24,25]$.

The mechanism of cyclic production to improve the displacement effect of vertical heterogeneity is the interlayer cross-flow caused by capillary force and additional pressure $[26,27]$, while the mechanism of cyclic production to improve the displacement effect of areal heterogeneity is the activation of the dead oil region by periodic change of pressure field. Due to the periodic production and shut-in, the existence of the additional pressure drop generates certain disturbance, which varies with the form, location, and size of the dead oil region. The remaining oil zone shrinks with the shut-in and restart of the production well. The macromechanism of the capillary force during the periodic production is to cause the fluid exchange between the higher permeability layer and low-permeability layer. And the micromechanism is to arouse the fluid exchange between closed pores, thin network channels, and coarse network channels [28]. The mechanism of additional pressure during the period production is very similar with cyclic injection, which includes two parts: one is the cross-flow of the oil and water from the lower permeability layers to the higher permeability layers at the initial stage of shut-in, and the other one is the cross-flow of the oil and water from the higher permeability layers to the lower permeability layers at the initial stage of restart.

Cyclic injection and production are the combination of cyclic injection at the injection well and cyclic production at the production well at the same time. It means that it not only generates additional pressure drop at the injection well region but also generates additional pressure drop at the production well region. The additional pressure drop between the higher and lower permeability layers causes the oil and water exchange and seeps into the seepage channels [29]. Thus, with the activation of the dead oil region by periodic variations of the additional pressure field, the capillary force effect and elastic effect are fully used. And finally, the water displacement effect of the formation with both the vertical heterogeneity and areal heterogeneity is improved.

Hydrodynamic methods like shut-in and restart process were greatly adopted and promoted in the oilfields, which achieved great improvements [19]. After the implement of these operations when the water cut of the production well achieved $98 \%$, either it was shut-in or tertiary recovery methods are conducted [15]. However, what will happen to the formation during the well shut-in? Has the production well or the reservoir really approach the economic limits of waterflooding? What the water cut of the production well changes if it is re-opened after a period of time? Is it still higher than $98 \%$ ? There are plenty of similar questions.

To answer the above questions, waterflooding experiments of the heterogeneous reservoir after well shut-in are conducted. The objective of this study is to evaluate the effect of shut-in and restart on the recovery of oil reservoir with high water cut and high heterogeneity. Firstly, a physical model with three different layers was simplified and made according to the physical characteristics of the potential reservoir. On this basis, waterflooding, first-time shut-in and restart, and second-time shut-in and restart were carried out. Cumulative oil recovery, water saturation distribution, and remaining oil saturation of three different layers before and after the well restart are obtained and analyzed. Besides, the macro- and micromechanisms of the shut-in and restart were studied. Moreover, some enlightenments of the study for the economic and effective development of the formation were presented accordingly. The novelty of this investigation is that the dynamic characteristics of shut-in and restart process in reservoirs with high water cut and strong heterogeneity are learned. In addition, we have made it clear that the process of shut-in and restart the reservoir has a significant impact on the redistribute of oil and water in the formation.

\section{Experiments}

The SD 3/7 block is mainly composed of the Yabus and Samaa groups, which include several sand layers. To simulate the reservoir heterogeneity of inner layer and its influence on water flooding, experiments based on the simplified physical model with the basic reservoir characteristics were conducted.

2.1. Materials. It should be noted that the reservoir temperature is $85.5^{\circ} \mathrm{C}$. However, the temperature resistance of the epoxy resin which cemented the model is very limited; thus, the actual experimental temperature is $55^{\circ} \mathrm{C}$. Oil sample was recombined with the crude oil and kerosene, and the viscosity is $1.25 \mathrm{MPa}$. The recombination is to reduce the oil viscosity. On the one hand, oil with higher viscosity will 
adhere to the electrode and affect the measurement results; on the other hand, the pressure-bearing capacity of the model is limited, and the injection pressure must not be too high. The salinity of the brine is $10000 \mathrm{mg} / \mathrm{L}$, and the viscosity is $0.554 \mathrm{MPa}$.

2.2. Apparatus. The plane geometry size of the model is 31 $\mathrm{cm} \times 31 \mathrm{~cm} \times 7.42 \mathrm{~cm}$, which consists of 3 layers with equal thickness and is suppressed by quartz sand and epoxy resin.

Different permeability values of the model are realized through compaction of sandstone with different particle sizes under different pressures in the seal mold. Then, as the pressure gradually increases, we need to monitor the gas permeability changes during compaction in real time. When permeability reached our desired value, we stopped adding pressure. From top to bottom, the permeabilities of the three layers are $100 \times 10^{-3} \mu \mathrm{m}^{2}, 450 \times 10^{-3} \mu \mathrm{m}^{2}$, and $1400 \times 10^{-3} \mu$ $\mathrm{m}^{2}$, respectively. For permeability test in each layer, we obtained it by injecting air at a constant pressure in a seal mold that can apply different pressures. For porosity test in each layer, we obtained it by saturating every layer with brine at a constant pressure in a seal mold. The model belongs to positive rhythm sedimentation. We place filter paper between two adjacent small layers to reduce the influence of the layer-to-layer interface. It means that two adjacent layers within the model are connected to each other. They can influence each other in the actual displacement process. At last, we used epoxy to seal the entire model. Honestly, the influence of layers in between is only reduced, not eliminated. So, we think that there is no interlayer between two adjacent layers. The variation coefficient is 0.72 . Table 1 shows the basic parameters of the physical model, which contains 137 pairs of electrodes. No electrode was located at the coordinates $(1,1),(1,7),(7,1),(7,7)$, and $(4,4)$, as is shown in Figure 1 . The resistance values are calculated by that of the adjacent coordinates.

Considering the big permeability difference, oil is easy to advance along the high-permeability layer. To fully saturate the model and truly simulate the original state of the reservoir, there are 19 wells placed in the model. Both general water injection and separate water injection can be well modelled. The well numbers of the layers from top to bottom are 5,9 , and 5 , which means both the five-spot pattern and ninespot pattern can be simulated. Figure 2 shows the schematic of the test.

2.3. Procedure. Calibration experiments are needed before the experiment. The oil and water are injected into the calibration model of each small layer in a certain proportion. When the pressure is stable, the average resistance of multiple electrodes is taken as the resistance value under the oil saturation. Then, the oil-water ratio was changed to get the resistance value at different oil saturations, so as to get the relationship curve between the resistance value and oil saturation.

Firstly, weight the model, and test the gas permeability and gas porosity. Then, evacuate the model, and test the pressure endurance and leakage. After that, saturate the model with brine, and test the pore volume (PV), water relative permeability, and single-phase water resistance.

Secondly, saturate the physical model with oil, and shut down the high-permeability layer, medium-permeability layer, and low-permeability layer one by one when it is fully saturated, test the brine production, and then obtain the irreducible water saturation. As is shown in Figure 1(d), we use switches installed from each small layer to control the opening and closing of each small layer. Since the entire model is sealed by epoxy resin, there is no leakage in a low-pressure environment. Age the model for 24 hours, and test the electrode resistance value when the brine and oil is stable in the model.

Thirdly, for the waterflooding process of the model, the three layers will produce at the same time. Then, close the injection and production valves of the high-permeability layer when no oil is produced in this layer. Similarly, close the injection and production valves of the media permeability layer and the low-permeability layer when no oil is produced, respectively. All the tests were conducted at a constant pressure of $0.3 \mathrm{MPa}$.

Finally, restart the production after half a month and five months, and keep the production until the water cut is over $98 \%$, respectively. Record the resistance value, oil production rate, and water production rate during the tests.

\section{Results and Discussion}

The heterogeneous model was water flooded for 1.24 HCPV (hydrocarbon pore volume) when the water cut is $98.03 \%$. Then, it was shut-in for half a month before restarting again. The experimental conditions are just the same as the original water flooding.

3.1. Water Cut. As is shown in Figure 3, for the original water drive, water breakthrough occurs after $0.2 \mathrm{HCPV}$; then, the water cut increases sharply to $80 \%$ at $0.3 \mathrm{PV}$ and $100 \%$ at $0.38 \mathrm{PV}$, respectively. It was labeled as the 1st stage. The production of both water and oil is mainly from the highpermeability layer. After that, the high-permeability layer is closed. Then, it enters the 2nd stage, in which the oil was mainly produced by the medium-permeability layer. The comprehensive water cut declined rapidly to $10 \%$ until $0.4 \mathrm{PV}$, then went back to $80 \%$ after $0.55 \mathrm{PV}$. Finally, the water cut increases gradually to $96 \%$ until $0.85 \mathrm{PV}$. It is concluded that cross-flow occurs between mediumpermeability layer and low-permeability layer, which results in this steady stage.

Similarly, the medium-permeability layer is closed. And the 3rd stage, which is mainly produced by lowpermeability layer, is achieved. The water cut decreases sharply to $5 \%$ and then increases rapidly until the production stage ends.

Half a month later, the first restart begins, and the water cut decreases a little bit initially and then changes smoothly. After 0.46 HCPV of water was injected (the accumulative volume is $1.70 \mathrm{HCPV}$ ), the water cut of production well is $96.61 \%$. 
TABLE 1: Basic parameters of the physical model.

\begin{tabular}{|c|c|c|c|c|c|}
\hline Type & $\begin{array}{c}\text { Geometry size }(\mathrm{cm}) \\
(\text { length } \times \text { width } \times \text { height })\end{array}$ & $\begin{array}{l}\text { Porous volume } \\
\qquad\left(\mathrm{cm}^{3}\right)\end{array}$ & $\begin{array}{c}\text { Porosity } \\
(\%)\end{array}$ & $\begin{array}{l}\text { Permeability } \\
\left(10^{-3} \mu \mathrm{m}^{2}\right)\end{array}$ & $\begin{array}{c}\text { Irreducible water } \\
\text { saturation }(\%)\end{array}$ \\
\hline \multirow{3}{*}{$\begin{array}{l}\text { Inner layer } \\
\text { heterogeneity }\end{array}$} & \multirow{3}{*}{$31 \times 31 \times 7.42$} & \multirow{3}{*}{1701.45} & & 100 & \multirow{3}{*}{37.245} \\
\hline & & & 23.85 & 450 & \\
\hline & & & & 1400 & \\
\hline
\end{tabular}

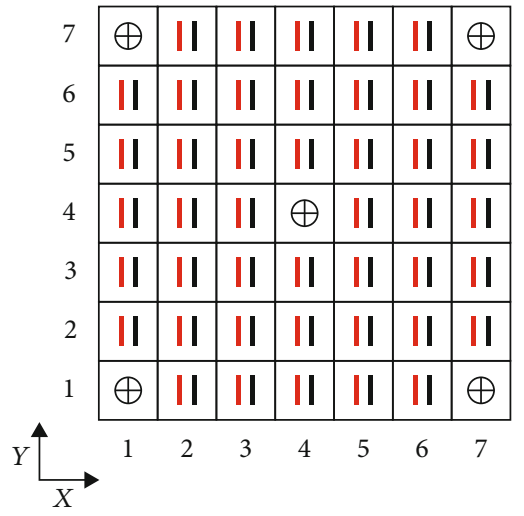

II Electrode

$\oplus$ Well

(a)

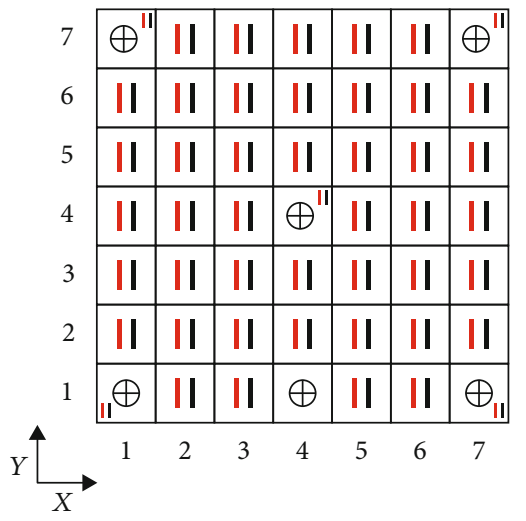

|| Electrode

$\oplus$ Well

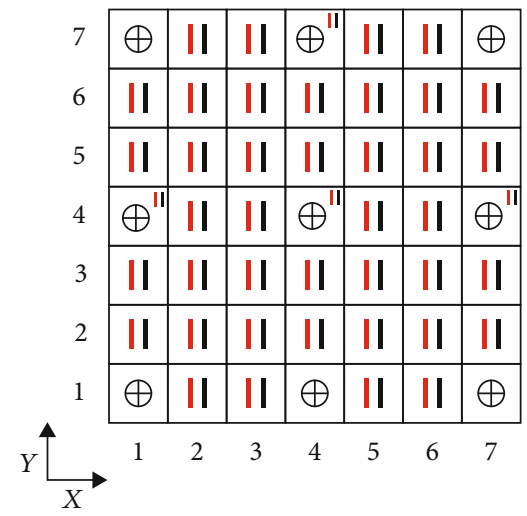

I| Electrode

$\oplus$ Well

(b)

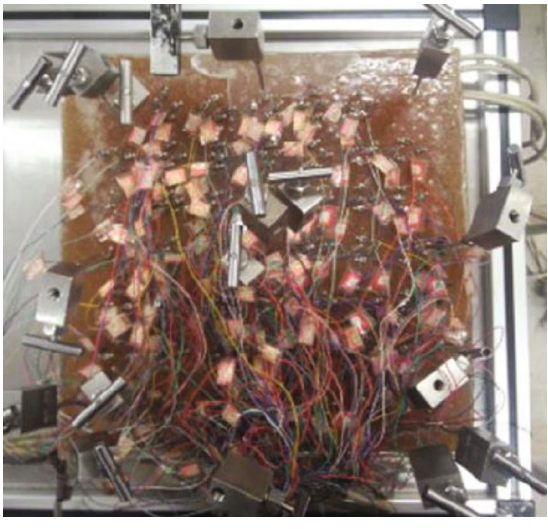

(c)

(d)

Figure 1: The distribution of the electrodes and wells injectors: well $(1,1)$; producers well $(7,7)$. (a) $k=100 \times 10-3 \mu \mathrm{m}^{2}$. (b) $k=450 \times 10$ $-3 \mu \mathrm{m}^{2}$. (c) $k=1400 \times 10-3 \mu \mathrm{m}^{2}$. (d) The model.

Then, the well was shut-in and restarted again after 5 months. The experimental conditions keep constant. The water cut of the 2 nd restart changes more significantly. At the beginning, the water cut is very high and then decrease with some oil production. The unsteady water cut after that is mainly caused by the cross-flow between different layers. After $0.68 \mathrm{HCPV}$ of water was injected (the accumulative volume is $2.38 \mathrm{HCPV}$ ), the water cut of production well is $99.12 \%$.
3.2. Oil Recovery. The oil recovery is the ratio of the production oil to the original oil in the model. Figure 4 shows the oil recovery changes with the injection volume. Obviously, for the original water flooding process, oil recovery was contributed by the high-, medium-, and lowpermeability layers one after another. Because of the cross-flow in between, the three different stages are not totally corresponding to the oil recovery of each layer. As is shown in Table 2, the 1st stage is mainly produced 

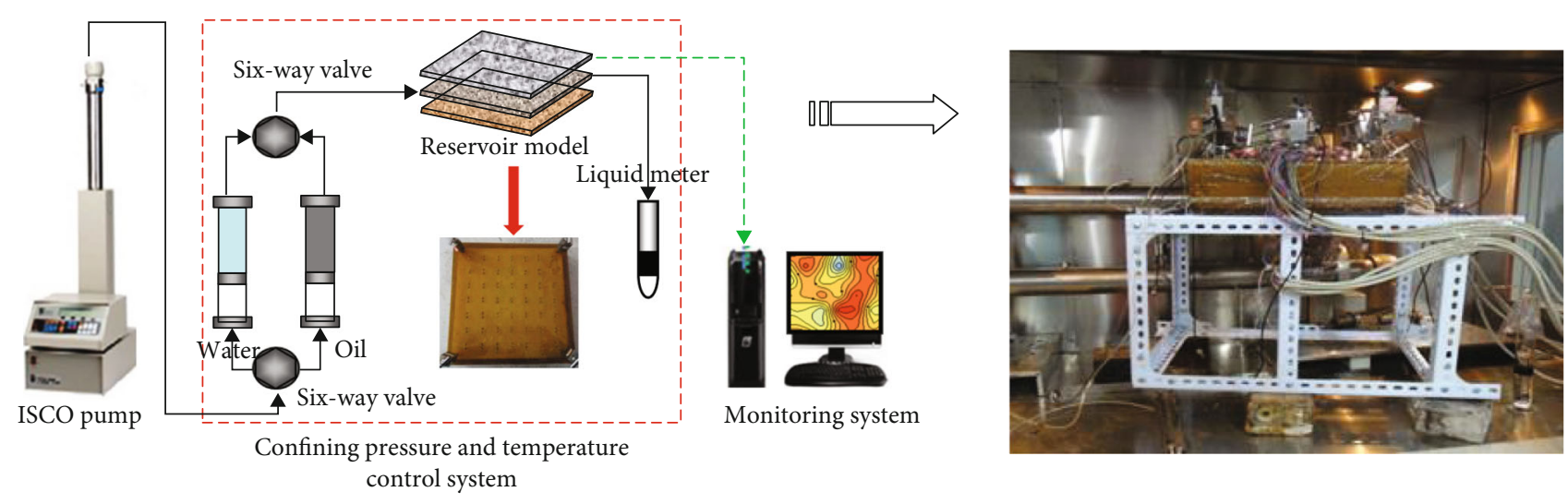

Figure 2: The schematic of the test.

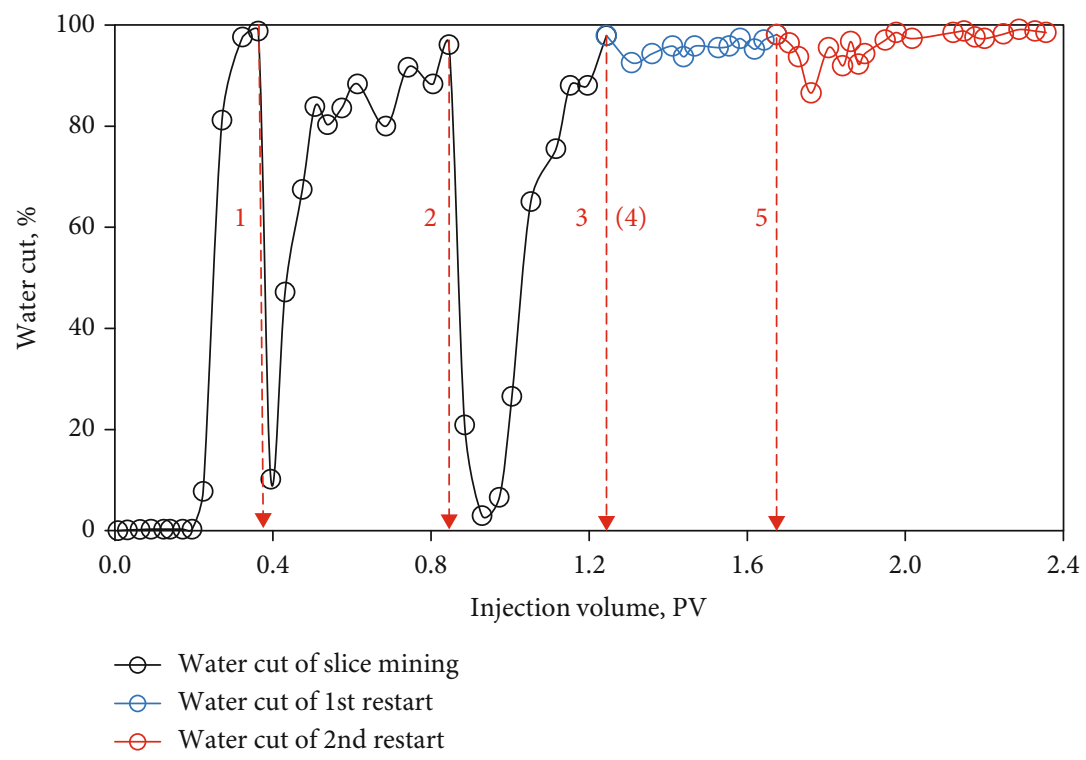

FIGURE 3: The variation law of water cut during the whole water flooding process (1, the whole oil layers are perforated; 2 , the perforated layers are closed; 3 , the perforated layers are closed; 4 , 1st restart; 5, 2nd restart).

from the high-permeability layer, and the injection volume and the oil recovery are $0.36 \mathrm{PV}$ and $37.03 \%$, respectively. The 2nd stage is mainly produced from the mediumpermeability layer, and the cumulative volume and the cumulative oil recovery are $0.84 \mathrm{PV}$ and $54.50 \%$, respectively. The 3rd stage is mainly produced from the lowpermeability layer, and the cumulative volume and the cumulative oil recovery are $1.24 \mathrm{PV}$ and $77.12 \%$, respectively. It is concluded that due to the cross-flow and gravitational differentiation, parts of the water flowing into the bottom high-permeability layer are from the top low- and medium-permeability layers near the injection well. The changing curves of the oil recovery are very similar, which increase slightly. It indicates that the remaining oil redistributes in the model during the shut-in periods, which results in the production of parts of the remaining oil.

3.3. Distribution of Oil Saturation. As is shown in Figure 5, after the water displacement process, the oil saturation of high-permeability layer is very low. Parts of the mediumpermeability layer are of low water cut and with high oil saturation, but the whole sweep efficiency of this layer is still very high. However, for the low-permeability layer, because of the interlayer interference and gravitational differentiation, both the overall displacement efficiency and sweep efficiency are very limited with large local difference. Obviously, it is the main potential area for the adjustment of the later development stage.

Figure 6 is the distribution of the remaining oil before first restart. It can be seen that after shutting in a period of time, parts of the oil and water redistribute in the model. Parts of the remaining oil in the corner which is difficult to displace diffuse towards the middle part. Both the remaining oil and water in the model converge locally.

Figure 7 presents the distribution of the remaining oil after first restart. Apparently, the water saturation of all three layers changes, especially for medium-permeability layer and low-permeability layer. In other words, parts of the 


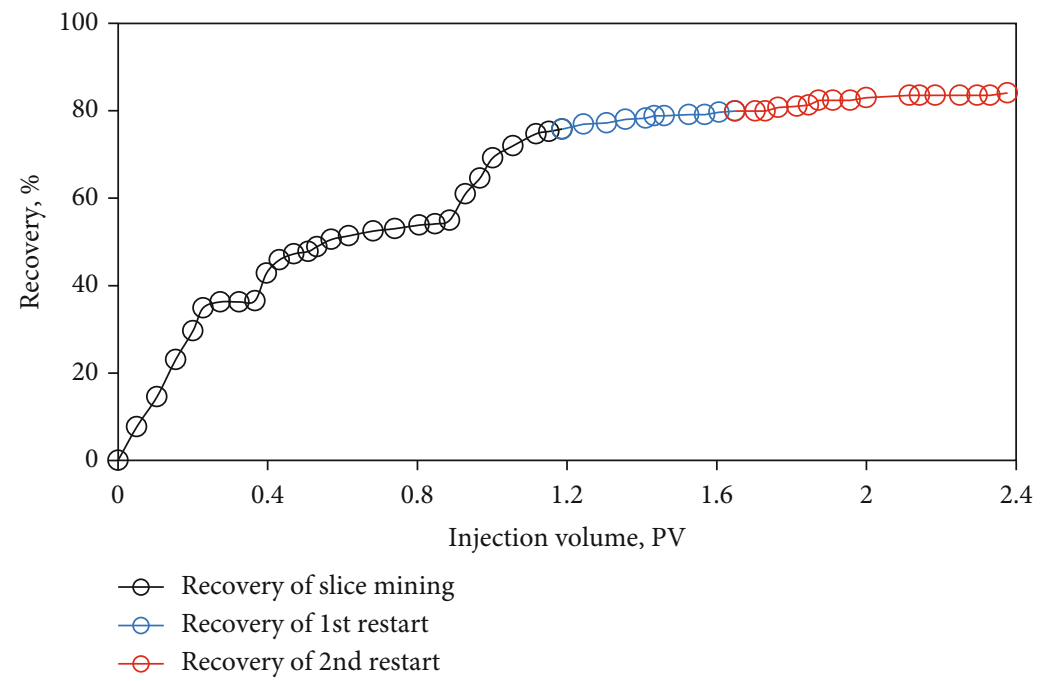

FIGURE 4: Oil recovery vs. injection volume.

TABLE 2: Oil saturation in different periods.

\begin{tabular}{lcccc}
\hline Stage & $\begin{array}{c}\text { Low permeability, } \% \\
\left(100 \times 10^{-3} \mu \mathrm{m}^{2}\right)\end{array}$ & $\begin{array}{c}\text { Medium permeability, } \% \\
\left(450 \times 10^{-3} \mu \mathrm{m}^{2}\right)\end{array}$ & $\begin{array}{c}\text { High permeability, \% } \\
\left(1400 \times 10^{-3} \mu \mathrm{m}^{2}\right)\end{array}$ & $\begin{array}{c}\text { Total oil recovery, } \\
\%\end{array}$ \\
\hline 1st & 97.94 & 43.98 & 11.54 & 36.54 \\
2nd & 85.55 & 18.66 & 3.21 & 54.95 \\
3rd & 43.22 & 8.31 & 0.79 & 75.82 \\
\hline
\end{tabular}

remaining oil after waterflooding can be displaced by shutting in for a period of time.

Similarly, Figure 8 shows oil saturation of all three layers after shutting in for 5 months. Parts of the oil and water redistribute in the model. Parts of the remaining oil in the corner which is difficult to displace diffuse towards the middle part. Both the remaining oil and water in the model converge locally. However, the remaining oil distribution for the 2nd restart is basically the same as that of the first time, as indicated in Figure 9. After the 1st restart process, there is less remaining oil left; thus, the change of the remaining oil saturation is not as significant as the initial. There are only very small parts of the oil diffuse into the middle part of the model.

\subsection{Phenomena of Restart Process}

3.4.1. Change of Pressure and Flowing Rate. The pressure of the model decreases gradually after shut-in for a period of time. Once the well was restarted, the flowing rate suddenly increases in the model, and then, the steady state breaks. The injection water shocks and crushes the oil in the pore channels. Thus, parts of the oil which is very difficult to produce were displaced, and the oil recovery increased.

3.4.2. Change of Water Cut and Oil Recovery. The increase of the oil recovery after restart mainly depends on two parts. Firstly, sweep efficiency increases with the expanding of water flooding. The enlargement of the flooding area is actu- ally the dynamic adjustment of the oil-water contact. For the initial stage of the restart process, the balance of the oil and water in the model was destroyed. With the continuous water displacement, water keeps moving forward, and some oil was produced until the new balanced reestablishment. In addition, some remaining oil drops in the water injection channels were taken away with the water production. During the enlargement of the waterflooding, the oil displacement did not cease in the waterflooding paths. Under the effect of hydrodynamics, the remaining oil was cut off and coalesces over and over again. Finally, some oil drops were displaced. Therefore, significant oil saturation gratitude forms between the upstream and downstream along the injection channels. Obviously, the remaining oil residuals at the channels and flows around the unflooded layers are very high.

3.4.3. Jamin Effect. During the process of the two-phase seepage flow, if one phase is dispersed as droplets in another phase, additional capillary resistance will generate when the droplets are deformed due to the pressure or move at the porous channels with the changing diameters. The phenomenon of water locked is named the Jamin effect. After water breakthrough, the two-phase seepage of oil and water forms, and the residual oil droplets or oil columns exist at the waterflooded pores. When affected by external forces or flows through pore throat, deformation occurs, and additional capillary pressure resistance generates; the accumulation of these forces will have great influence on the waterflooding process. During the test, the water is injected at a constant pressure; 

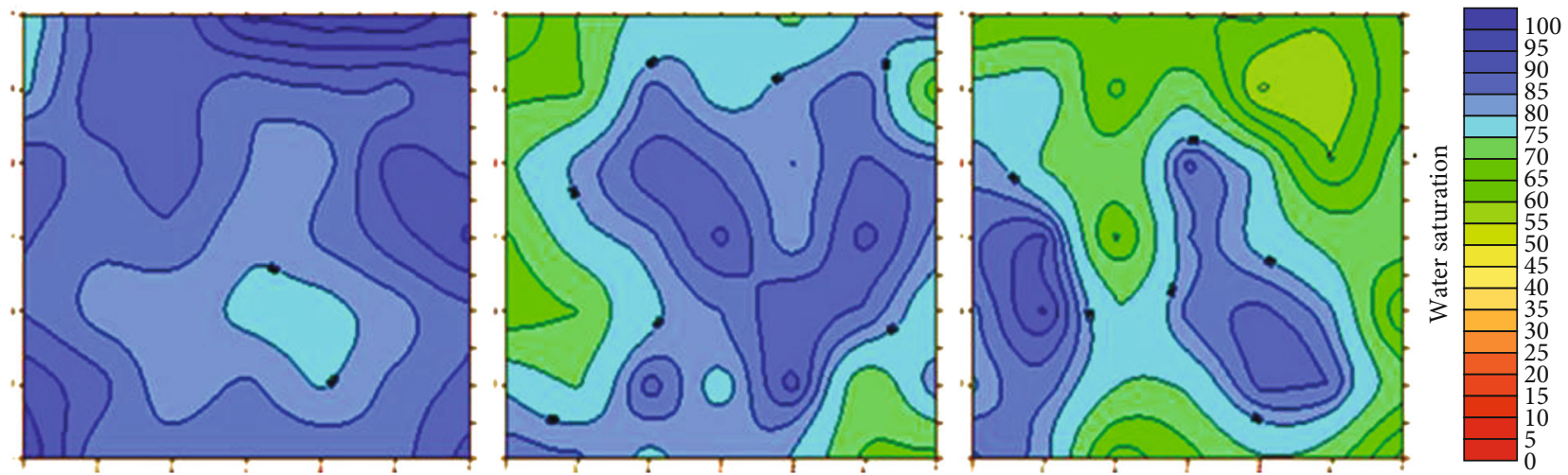

FIGURE 5: The distribution of the remaining oil after water displacement (the permeability of the layers from left to right: 1400, 450, and 100, $\left.\times 10^{-3} \mu \mathrm{m}^{2}\right)$.
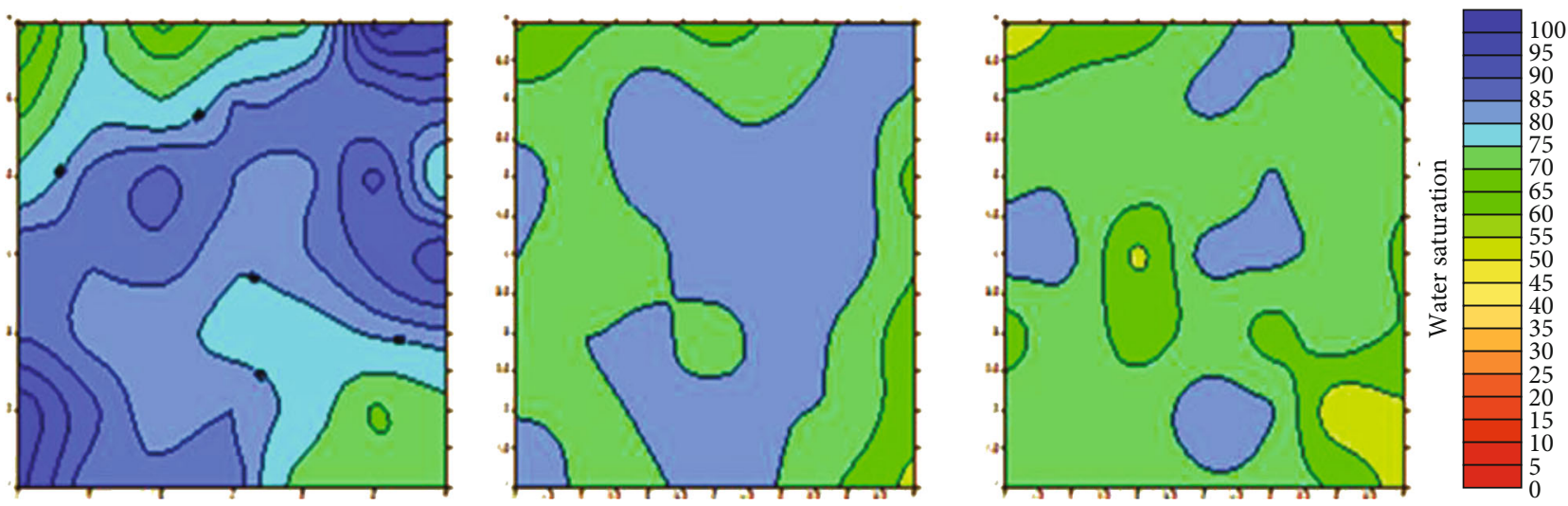

Figure 6: The distribution of the remaining oil before the first restart (the permeability of the layers from left to right: 1400, 450, and $\left.100, \times 10^{-3} \mu \mathrm{m}^{2}\right)$.
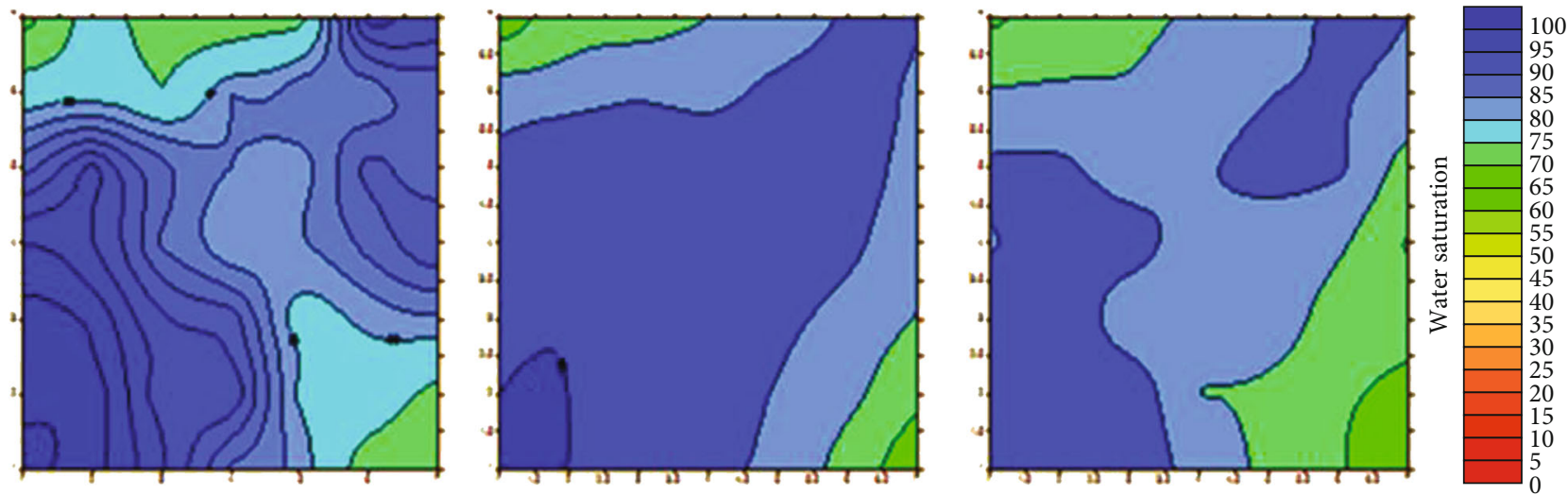

Figure 7: The distribution of the remaining oil after the first restart (the permeability of the layers from left to right: 1400 , 450 , and $\left.100, \times 10^{-3} \mu \mathrm{m}^{2}\right)$.

therefore, with the resistance of waterflooding, the flowing rate gets smaller and smaller. Besides, the retentions also occur for some deformed oil drops, which cannot migrate out of the model; it is also the result of the Jamin effect. In sum, the existence of Jamin effect not only stops the movement of some oil drops but also deadlocks some of the displacement channels. Consequently, the waterflood- ing effect is greatly influenced, and the production declines greatly.

Therefore, in the actual production process, according to the experimental phenomenon, firstly, we should open the injection well and then the production well. Secondly, we suggest opening the wells in the middle of the reservoir first and then the wells at the corner. Thirdly, we open the 

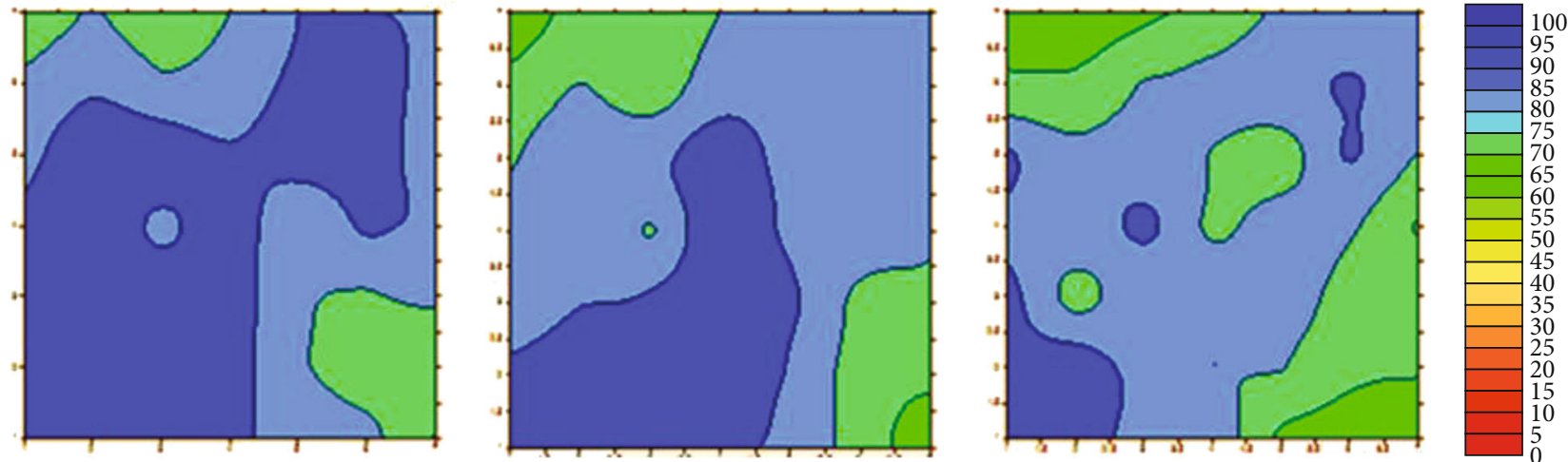

Figure 8: The distribution of the remaining oil before the second restart (the permeability of the layers from left to right: 1400,450 , and 100, $\left.\times 10^{-3} \mu \mathrm{m}^{2}\right)$.
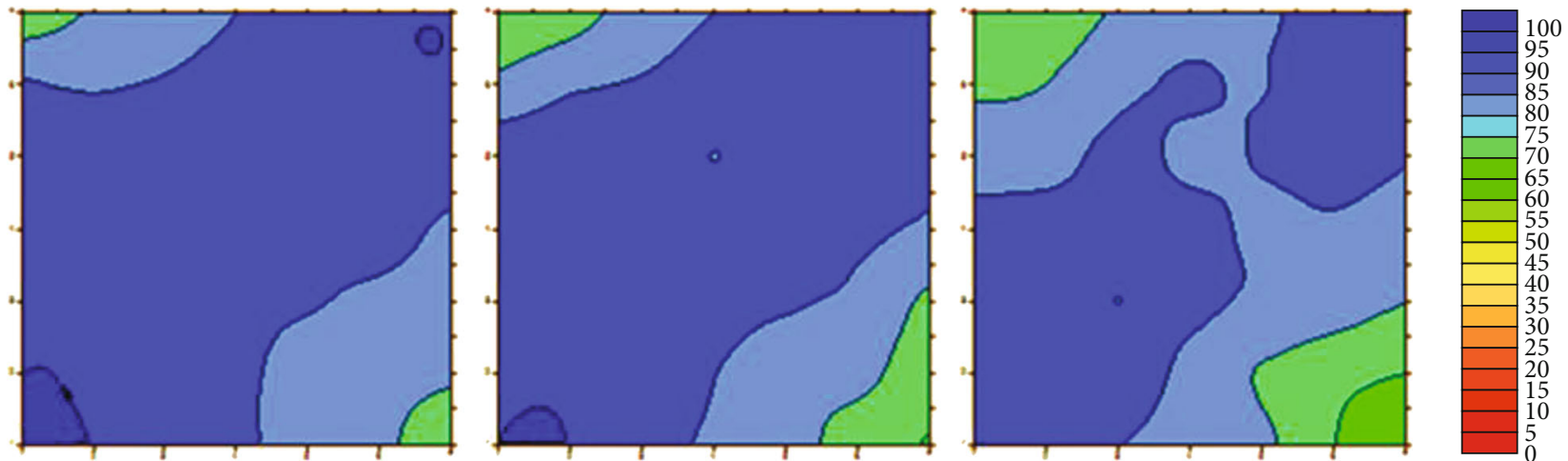

Figure 9: The distribution of the remaining oil after the second restart (the permeability of the layers from left to right: 1400, 450, and 100, $\left.\times 10^{-3} \mu \mathrm{m}^{2}\right)$.

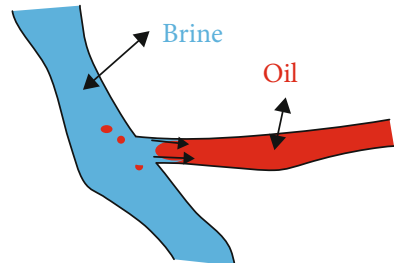

FiguRE 10: The capillary force effect for water wet reservoir.

injection well with a lower flowing rate, while we open the production well with a higher flowing rate. On the one hand, the pressure in the model rises, the seepage velocity is very high when it is opened, and the injected water hits the crude oil in the pores, making the crude oil that is difficult to recover during the original water flooding easier to recover. On the other hand, remove the crude oil at the corners effectively, reduce water cut, and prolong breakthrough time to increase oil recovery.

3.5. Restart Mechanism. Before the shut-in of the well, the oil distributes mainly as a continuous oil phase, oil segments, or oil drops scattered in some pores. However, most of the oil was bypassed or trapped due to the capillary force during water displacement. After that, the shut-in of well decreases the flowing pressure difference, the movement of the liquids in the model slows down gradually and ceases finally. However, for the reservoirs with edge and bottom water, oil and water will still move towards the bottom of the production well due to the existence of the pressure funnel. As time goes on, under the effect of capillary pressure, there exists microscopic migration of water and oil. For oil-wet reservoirs, the redistribution of oil and water will be much slower. For reservoirs with inner layer heterogeneity, the cross-flow in between is a beneficial mechanism, which pushes the oil from the low-permeability layer to higher permeability layers. Specifically, based on the shut-in and restart dynamic test, three mechanisms can be concluded as follows.

3.5.1. Capillary Force Effect. Laboratory test shows that the movement velocity of the oil-water contact in two parallel pore channels with different radius is different. One contact arrived at the junction first and then continued to move along the channel, but the other one was trapped in the channel. Thus, if the pressure is high enough, the flowing rate in the bigger pore channel is higher while the oil in the smaller channel retained. Such water flows along the bigger channels to displace the oil in the bigger channels, while the oil in the smaller channels is left untapped during the water flooding. However, for the water wet reservoir, if the pressure is too low, the capillary force dominates and controls the flowing 


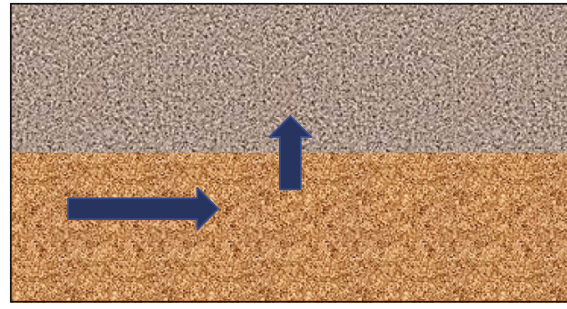

(a)

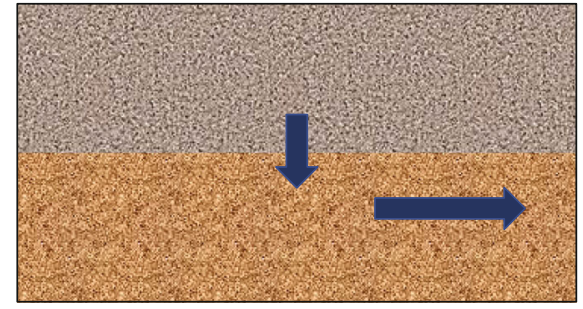

(b)

FIGURE 11: Flow directions during pulse water injection. (a) Pressure increase stage. (b) Pressure decrease stage.

rate and direction of the oil-water contact, and then, the oil in the bigger pore channel was retained [30]. For example, as is shown in Figure 10, the pressure is low, and the capillary force predominates during the shut-in. Depending on the capillary force, water gradually enters the small pore to displace the oil.

3.5.2. Plug Removal of Pressure Difference Mechanism. Firstly, enhance the pressure of the oil layer, inject fluids, and then decrease the injection rate to reduce the pressure after a period of time. It has an impulsive effect on the oil layer. Water injection and pressure increase are highest in the high-permeability layer, which results in the flowing of the oil and water into the lower permeability layers under the additional pressure difference [31]. On the contrary, when the water injection ceased and the formation pressure decreased, the pressure of the high-permeability layer decreased fastest, and then, parts of the oil and water flow towards the higher permeability layer under the effect of this backward additional pressure difference and are then produced on the next pressure increase process, as is shown in Figure 11.

3.5.3. Elastic Displacement Mechanism. Impulsive effect of the cyclic variation of the water injection pressure to the oil layer can enhance the elastic energy of the oil formation [32]. Pressure decrease can degas the remaining oil locked at the pore walls and throats, then release the elastic energy, and displace the oil in the pore channels.

\section{Conclusion}

Based on the shut-in and restart test, the following conclusions can be drawn:

(1) For developing strong heterogeneity reservoirs, appropriate developing plan such as layering mining and cyclic waterflooding should be designed. The shut-in and restart test showed that closing the waterflooded layer is beneficial for enlarging the sweep efficiency and effectively employing the remaining oil

(2) Typically, the production wells are closed when the water cut attained $98 \%$. However, under the effect of the capillary force and gravitational differentiation, oil and water will redistribute in the reservoir. Parts of the remaining oil can be produced again. In the shut-in and restart test, with two times restart process, the cumulative recovery increased by $7 \%$ after the water cut of the production well attained $98 \%$

(3) During the shut-in period, oil and water in all three layers redistribute. Oil in the intermediate- and lowpermeability layers mainly moves towards highpermeability layer. It can be seen that the effect of capillary force is much more obvious than that of gravitational differentiation. The redistribution of the oil and water, the fluctuation of the pressure difference, and the rebuild of the flow path, which produces parts of the bypassed oil, are the main mechanisms of the recovery enhancement by shutin and restart

(4) The shut-in and restart process are very similar to cyclic water injection. It can decrease the water cut and increase the oil production to some extent. However, the shut-in period is adjustable. It should be noted that although the shut-in and restart process indeed prolonged the waterflooding development, the enhanced oil recovery is only $7 \%$ in the test, which means that simply replying on the oil and water distribution under static conditions cannot greatly enhance the oil recovery

(5) For the working schedule after restart, it is recommended as follows: Firstly, open the injection well first, then the production well. Secondly, open the wells in the middle of the reservoir, then the wells at the corner. Thirdly, open the injection well with lower flowing rate, while open the production well with higher flowing rate.

\section{Data Availability}

The data used to support the findings of this study are included within the article.

\section{Conflicts of Interest}

The authors declare that they have no known competing financial interests or personal relationships that could have appeared to influence the work reported in this paper. 


\section{References}

[1] T. C. Laforce and F. M. Orr, "Development of gas/oil miscibility in water and gas injection," in SPE Annual Technical Conference and Exhibition, Denver, CO, USA, September 2008.

[2] L. L. Brundred and L. L. Brudred, "Economics of water flooding," Journal of Petroleum Technology, vol. 7, no. 1, pp. 12-17, 1955.

[3] M. Cimic, "Russian mature fields redevelopment," in SPE Russian Oil and Gas Technical Conference and Exhibition, Moscow, Russia, October 2006.

[4] A. Collins, "Enhanced-oil-recovery injection waters," in SPE International Oilfield and Geothermal Chemistry Symposium, La Jolla, California, June 1977.

[5] A. M. S. Sarem, "Secondary and tertiary recovery of oil by MCCF process," in SPE California Regional Meeting, San Francisco, California, April 1974.

[6] L. O. Masoner, "Decline analysis relationship to relative permeability in secondary and tertiary recover," Proceedings of the National Academy of Sciences of the United States of America, vol. 47, no. 8, pp. 1141-1149, 1961.

[7] M. Husband, D. Ohms, H. Frampton et al., "Results of a threewell waterflood sweep improvement trial in the Prudhoe Bay Field using a thermally activated particle system," in SPE Improved Oil Recovery Symposium, Tulsa, OK, USA, April 2010.

[8] N. Morrow and J. Buckley, "Improved oil recovery by lowsalinity waterflooding," Journal of Petroleum Technology, vol. 63, no. 5, pp. 106-112, 2011.

[9] H. Kazemi and L. S. Merrill, "Numerical simulation of water imbibition in fractured cores," Society of Petroleum Engineers Journal., vol. 19, no. 3, pp. 175-182, 1979.

[10] L. K. Thomas, T. N. Dixon, and R. G. Pierson, "Fractured reservoir simulation," Society of Petroleum Engineers Journal., vol. 23, no. 1, pp. 42-54, 1983.

[11] P. Sigmund, H. Sharma, D. Sheldon, and K. Aziz, "Rate dependence of unstable waterfloods," SPE Reservoir Engineering, vol. 3, no. 2, pp. 401-409, 2013.

[12] J. Groenenboom, S.-W. Wong, T. Meling, R. Zschuppe, and B. Davidson, "Pulsed water injection during waterflooding," in SPE International Improved Oil Recovery Conference in Asia Pacific, Kuala Lumpur, Malaysia, October 2003.

[13] L. E. Cuiec, B. J. Bourbiaux, and F. Kalaydjian, "Oil recovery by imbibition in low-permeability chalk," SPE Formation Evaluation, vol. 9, no. 3, pp. 200-208, 1994.

[14] J. E. Smith, "Performance of 18 polymers in aluminum citrate colloidal dispersion gels," in SPE International Symposium on Oilfield Chemistry, San Antonio, Texas, February 1995.

[15] P. H. Krumrine and S. D. Boyce, "Profile modification and water control with silica gel-based systems," in SPE Oilfield and Geothermal Chemistry Symposium, Phoenix, Arizona, April 1985.

[16] G. Zangl and R. Hermann, "Waterflood pattern optimization using genetic algorithms with multi-tank material balance," in SPE Annual Technical Conference and Exhibition, Houston, Texas, September 2004.

[17] X. Anzhu, M. Longxin, Z. Lun et al., "A new strategy of well pattern design and adjustment to enhance production of vertical multiple reservoirs," in SPE Kuwait International Petroleum Conference and Exhibition, Kuwait City, Kuwait, December 2012.
[18] D. M. Wang, J. C. Cheng, and W. U. Wen-Xiang, "Combining small well spacing with polymer flooding to improve oil recovery of marginal reservoirs," in SPE/DOE Symposium on Improved Oil Recovery, Tulsa, OK, USA, April 2006.

[19] X. Pei, Z. Yang, B. Li, and L. Yaning, "History and actuality of separate layer oil production technologies in Daqing Oilfield," in International Oil \& Gas Conference and Exhibition in China, Beijing, China, December 2006.

[20] C. H. Wu, B. A. Laughlin, and M. Jardon, "Infill drilling enhances waterflood recovery," Journal of Petroleum Technology., vol. 41, no. 10, pp. 1088-1095, 1989.

[21] H. Liu, Z. Wang, and G. Xu, "Application and prospect of fine water-plugging technology in high water cut period," in SPE Asia Pacific Improved Oil Recovery Conference, Kuala Lumpur, Malaysia, October 1999.

[22] W. F. Zhou, S. J. Zhang, L. Yu, Q. Zhou, L. Shi, and C. R. Liu, "The integrated technology of water detection, shutoff and regulating separate layer production in the oil recovery process," in Nigeria Annual International Conference and Exhibition, Abuja, Nigeria, July 2011.

[23] A. Zaitoun, N. Kohler, D. Bossie-Codreanu, and K. Denys, "Water shutoff by relative permeability modifiers: lessons from several field applications," in SPE Annual Technical Conference and Exhibition, Houston, Texas, October 1999.

[24] L. F. Elkins and A. M. Skov, "Cyclic water flooding the Spraberry utilizes "end effects" to increase oil production rate," Journal of Petroleum Technology., vol. 15, no. 8, pp. 877-884, 1963.

[25] L. M. Surguchev, N. H. Giske, L. Kollbotn, and A. Shchipanov, "Cyclic water injection improves oil production in carbonate reservoir," Journal of Biological Chemistry, vol. 286, no. 4, pp. 3129-3138, 2008.

[26] D. R. Parrish, C. B. Pollock, and F. F. Craig, "Evaluation of COFCAW as a tertiary recovery method, Sloss field, Nebraska. SPE of AIME mid-continent sect," Improved Oil Recovery; (United States), vol. spe-3777, no. 6, pp. 676-686, 1974.

[27] F. Ghaddab, K. Kaddour, M. Tesconi, A. Brancolini, C. Carniani, and G. Galli, "El Borma-bright water-tertiary method of enhanced oil recovery for a mature field," in SPE Production and Operations Conference and Exhibition, Tunis, Tunisia, June 2010.

[28] A. S. L. Vaz, P. G. Bedrikovetsky, C. J. A. Furtado, A. G. Siqueira, and A. L. S. de Souza, "Effects of residual oil on reinjection of produced water," in SPE Europec/EAGE Annual Conference and Exhibition, Vienna, Austria, June 2006.

[29] A. A. Shchipanov, L. M. Surguchev, and S. R. Jakobsen, "Improved oil recovery by cyclic injection and production," in SPE Russian Oil and Gas Technical Conference and Exhibition, Moscow, Russia, October 2008.

[30] F. J. Rosado-Vazquez, E. Rangel-German, and F. R. De La Garza, "Analysis of capillary, gravity and viscous forces effects in oil/water displacement," in International Oil Conference and Exhibition in Mexico, Veracruz, Mexico, June 2007.

[31] G. Yang, Z. Honglan, L. He, Y. Jianwen, Y. Qinghai, and S. Fuchao, "Technique of water control and oil recovery based on water plugging combined with fracturing in low permeability and high water cut oilfield," in SPE Asia Pacific Oil and Gas Conference and Exhibition, Jakarta, Indonesia, October 2013.

[32] D. Wang, J. Cheng, Q. Yang, G. Wenchao, L. Qun, and F. Chen, "Viscous-elastic polymer can increase microscale displacement efficiency in cores," in SPE Annual Technical Conference and Exhibition, Dallas, Texas, October 2000. 